\title{
Beneficios clínicos y funcionales de agregar teofilina a la terapia inhalatoria con broncodilatadores de acción corta en pacientes con enfermedad pulmonar obstructiva crónica
}

\author{
Jorge Dreyse $D$ a, Felipe Silva $D$ a, O rlando Díaz $P$, \\ Gisella Borzone T, Carmen Lisboa B. \\ Clinical and functional benefits of adding \\ theophylline to a standard treatment with \\ short acting bronchodilators in patients \\ with COPD
}

Background: Although theophylline is considered a third line bronchodilator drug for the treatment of chronic obstructive pulmonary disease (COPD), it is widely used in Chile, because it is administered orally and has a moderate cost. Aim: To evaluate if theophylline adds clinical and/or functional benefits when associated to standard recommended inhaled bronchodilator therapy. Subjects and methods: Thirty-eight stable COPD patients who accepted to participate in the study approved by the Ethics Committee of our institution were studied. Using a randomized double-blind placebo-controlled study, theophylline (250 mg) or placebo was administered twice a day for 15 days in addition to inhaled salbutamol and ipratropium bromide. Prior to and at the end of the study, patients underwent: a) a spirometry to evaluate changes in dynamic pulmonary hyperinflation using slow vital capacity (SVC) and inspiratory capacity (IC), b) the 6 min walking distance (6 MWD); and c) measurement of maximal inspiratory and expiratory pressures. Dyspnea and quality of life (QoL) were evaluated using appropriate questionnaires. Results: Compared to placebo, patients on theophylline showed significant increases in SVC $(p=0.014)$, IC $(p=0.002)$, and 6 MWD $(p=0.005)$. They also experienced an improvement in dyspnea ( $\mathrm{p}=0.042)$ and $\mathrm{QoL}(\mathrm{p}=0.011)$. All patients improved at least one of these parameters with 53\% of the patients showing an improvement in 3 or more. Conclusions: Our results indicate that adding theophylline to standard treatment with inhaled bronchodilators provides additional benefits in stable COPD patients by reducing dynamic pulmonary hyperinflation, improving exercise tolerance, dyspnea and QoL (Rev Méd Chile 2005; 133: 1211-9).

(Key Words: Bronchodilator agents; Pulmonary disease, chronic obstructuve; Theophylline; Xanthines)

Recibido el 14 de diciembre, 2004. Aceptado el 3 de junio, 2005.

Proyecto de Inmersión de Verano 2004, Dirección de Investigación, Facultad de Medicina Pontificia Universidad Católica de Chile y Fondecyt 1010993.

Departamento de Enfermedades Respiratorias, Pontificia Universidad Católica de Chile, Santiago, Chile.

aAlumno de la Carrera de Medicina, PUC.

Correspondencia a: Dra Carmen Lisboa B. Marcoleta 345, 4ํㅜㄴ

piso. Santiago, Chile. Fax: 6335255. E-mail: clisboa@med.puc.cl 
La teofilina es una droga broncodilatadora empleada desde hace años en el tratamiento de la enfermedad pulmonar obstructiva crónica (EPOC). En la actualidad es considerada de tercera línea debido al niesgo de toxicidad cuando se emplea en dosis elevada, a la interacción con otras drogas y, fundamentalmente, a la disponibilidad de broncodilatadores de acción prolongada por vía inhalatoria que son eficaces en el control de los síntomas ${ }^{1,2}$. A diferencia de lo que ocurre con otras drogas broncodilatadoras de acción prolongada ${ }^{3,4}$, los estudios recientes acerca de la eficacia de la teofilina como broncodilatador único o asociado son escasos ${ }^{4,5}$.

$\mathrm{Si}$ bien distintos autores han demostrado que los broncodilatadores, tanto ß2-adrenérgicos como anticolinérgicos, disminuyen la hiperinflación pulmonar dinámica (HPD), uno de los factores que más contribuye a la disnea de ejercicio en pacientes con EPOC ${ }^{6-10}$, existen escasas publicaciones sobre el efecto de la teofilina en la HPD, con resultados contradictorios ${ }^{11-13}$.

Se ha demostrado que su asociación con una droga broncodilatadora por vía inhalatoria, produce una mejoría clínica adicional ${ }^{5,14-16}$. Sin embargo, la asociación de teofilina de acción retardada con la mezcla de salbutamol y bromuro de ipratropio ha mostrado resultados variables ${ }^{13,17,18}$.

El propósito del presente estudio fue evaluar con métodos simples el efecto de adicionar teofilina a un tratamiento broncodilatador con salbutamol y bromuro de ipratropio sobre la HPD, la obstrucción bronquial, la disnea, la capacidad de ejercicio, la calidad de vida y la fuerza muscular respiratoria en pacientes con EPOC moderada a muy grave, de acuerdo a la clasificación de GOLD (Global Initiative for Chronic Obstructive Lung Disease) ${ }^{1}$.

\section{MATERIAL Y MÉTODOS}

Pacientes. Se incorporó a 41 pacientes consecutivos, portadores de EPOC, ex fumadores de más de 20 paquetes año, en control en nuestro departamento, estables al momento del estudio, con un $\mathrm{VEF}_{1}$ inferior a $60 \%$ del teórico y sin antecedentes de intolerancia a teofilina. Todos aceptaron participar en el estudio y firmaron un acta de consentimiento informado aprobado por el comité de ética de nuestra institución.
Diseño. Se realizó un estudio prospectivo y doble ciego en que los pacientes fueron asignados a recibir aleatoriamente teofilina o placebo durante 15 días. La dosis de teofilina (Elixine ${ }^{\circledR}$ ) fue de 250 mg cada $12 \mathrm{~h}$. Aquellos que estaban usando teofilina ( $\mathrm{n}=17)$, la suspendieron 7 días antes del inicio del estudio $\mathrm{y}$, a partir del reclutamiento todos los pacientes fueron tratados con salbutamol $(240 \mu \mathrm{g})$ y bromuro de ipratropio $(40 \mu \mathrm{g})$ (Combivent ${ }^{\circledR}$ ) cada seis horas. Se les solicitó anotar en una hoja de control la hora de ingestión de la teofilina o el placebo, los posibles efectos adversos y el empleo del broncodilatador inhalatorio en dosis mayor a la indicada (medicación de rescate). Cuatro pacientes (2 de cada grupo) usaban, además, corticoides por vía inhalatoria y otros tres (2 del grupo de estudio y uno del grupo placebo), prednisona oral 5 a $10 \mathrm{mg} /$ día.

Las evaluaciones se realizaron tanto al inicio como en el día 15 del protocolo. Estas últimas se realizaron entre 3 y $8 \mathrm{~h}$ después de la dosis matinal de teofilina o placebo.

Mediciones. El estudio funcional incluyó una espirometría basal y luego de administrar $200 \mu \mathrm{g}$ de salbutamol en aerosol, empleando un espirómetro Spiro Analizer ST 250 (Fukuda Sanghio, Japón) siguiendo las normas de la Asociación Americana de Tórax ${ }^{19}$. Para los resultados se analizaron los cambios en $\mathrm{CVF}, \mathrm{VEF}_{1}$, capacidad vital lenta (CVL) y capacidad inspiratoria (CI). Los cambios en la HPD se evaluaron a través de las variaciones de la CVL y de la CI, los que se expresaron como valores absolutos y como porcentaje del valor teórico. Se consideró clínicamente significativo un aumento $\geq 10 \%$ del valor teórico según lo propuesto por la Sociedad Europea de Enfermedades Respiratorias ${ }^{20}$. El efecto sobre la fuerza muscular respiratoria se evaluó a través de las presiones inspiratorias y espiratorias máximas $^{21}$.

El estudio clínico incluyó: a) la capacidad de ejercicio evaluada con la prueba de caminata en 6 minutos, b) la disnea durante las actividades de la vida diaria y c) la calidad de vida. En la prueba de caminata en 6 minutos, además de la distancia recorrida (DR6) ${ }^{22}$ se registró el grado de disnea y fatiga de extremidades empleando la escala de Borg $^{23}$, la $\mathrm{SpO}_{2}$ y la frecuencia cardíaca. Todos los pacientes estaban familiarizados con esta prueba. 
Se consideró clínicamente significativo un aumento de la DR6 $>30 \mathrm{~m}^{24}$. La magnitud de la disnea se evaluó con la escala del Medical Research Council de Inglaterra $(\mathrm{MRC})^{25}$ que tiene una puntuación de 1 a 5 , donde 1 significa presentar disnea sólo con ejercicios extenuantes y 5 , disnea que impide salir del domicilio o que se presenta al vestirse 0 desvestirse. Se consideró clínicamente significativa una disminución de 1 punto en dicha escala. La calidad de vida se midió con el cuestionario específico para enfermedades respiratorias crónicas (Chronic Disease Respiratory Questionnaire $)^{26}$. Se consideró clínicamente importante un cambio de al menos 0,5 puntos en el puntaje global o en cada uno de los cuatro dominios del cuestionario 27 .

Los niveles plasmáticos de teofilina se midieron $6 \mathrm{~h}$ después de la dosis matinal el duodécimo día del estudio. Los resultados de dicho examen sólo se conocieron una vez finalizado el protocolo.

Estadística. Los resultados se expresan como promedios $\pm 1 \mathrm{DE}$. Para comparar los cambios obtenidos en el grupo experimental con los del grupo placebo se empleó la prueba t de Student para muestras no pareadas. La prueba de chicuadrado se utilizó para conocer la proporción de respondedores según los criterios funcionales y clínicos mencionados. Se consideró significativo un valor de $\mathrm{p}<0,05$.

\section{RESULTADOS}

Tres de los 41 pacientes fueron excluidos del estudio y se retiraron antes de completar el protocolo: uno antes de las mediciones basales, por acentuación de los síntomas tras suspender la teofilina; otro, al segundo día del estudio, por presentar náuseas y vómitos debido a que ingirió equivocadamente el medicamento cada $8 \mathrm{~h}$; el tercero, que estaba recibiendo placebo, por cumplir irregularmente con las indicaciones. La Tabla 1 muestra las características antropométricas y funcionales de los 38 pacientes al inicio del estudio. No hubo diferencias significativas entre las características de los grupos.

Los niveles plasmáticos de teofilina alcanzaron un promedio de 13,5 $\pm 4,8 \mathrm{mg} / \mathrm{l}$. Sólo dos enfermos del grupo tratado sobrepasaron $20 \mathrm{mg} / \mathrm{l}$ (Figura 1). No se observó relación entre el peso corporal y los niveles plasmáticos alcanzados $(\mathrm{r}=0,26 ; \mathrm{p}>0,1)$.

\section{Tabla 1. C aracterísticas antropométricas y funcionales de los pacientes con EPO C de los dos grupos estudiados}

\begin{tabular}{|lcc|}
\hline & $\begin{array}{c}\text { Placebo } \\
\mathrm{n}=19\end{array}$ & $\begin{array}{c}\text { Teofilina } \\
\mathrm{n}=19\end{array}$ \\
\hline Edad, años & $69 \pm 8$ & $66 \pm 9$ \\
Sexo, F/M & $3 / 16$ & $3 / 16$ \\
Talla, m & $164 \pm 7$ & $164 \pm 8$ \\
Peso, kg & $63 \pm 14$ & $65 \pm 14$ \\
CVL, \% teórico & $94 \pm 21$ & $92 \pm 24$ \\
CVF, \% teórico & $83 \pm 18$ & $83 \pm 23$ \\
VEF,$\%$ teórico & $35 \pm 10$ & $33 \pm 8$ \\
DR6, \% teórico & $82 \pm 11$ & $75 \pm 19$ \\
Disnea (MRC) & $3,1 \pm 1,1$ & $3,6 \pm 1,1$ \\
\hline
\end{tabular}

Los valores representan promedios $\pm 1 \mathrm{DE}$. $\mathrm{F}=$ femenino; $\mathrm{M}=$ masculino; $\mathrm{CVL}=$ capacidad vital lenta; $\mathrm{CVF}=$ capacidad vital forzada; $\mathrm{VEF}_{1}=$ volumen espiratorio forzado en un segundo; DR6=distancia recorrida en 6 min; MRC=escala de disnea del British Medical Research Council. No se observó diferencias significativas entre los grupos.

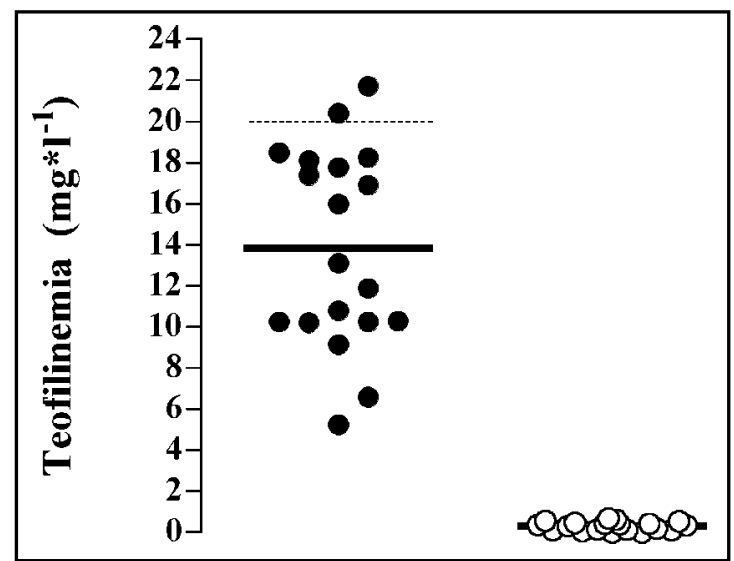

FIgURA 1. Niveles plasmáticos individuales de teofilina el duodécimo día del estudio en el grupo tratado con teofilina (círculos negros) y en el grupo placebo (círculos vacíos). La línea continua corresponde al promedio y la línea discontinua señala el nivel de $20 \mathrm{mg} / \mathrm{l}$. 
La Figura 2 muestra los cambios en valores absolutos y como porcentaje del valor teórico observados con teofilina o placebo de la CI, CVL, CVF y $\mathrm{VEF}_{1}$ en relación a los valores observados antes del tratamiento. La teofilina comparada con placebo produjo un aumento significativo de la CI y de la CVL La proporción de pacientes que aumentó la CI y la CVL $\geq 10 \%$ fue también significa-
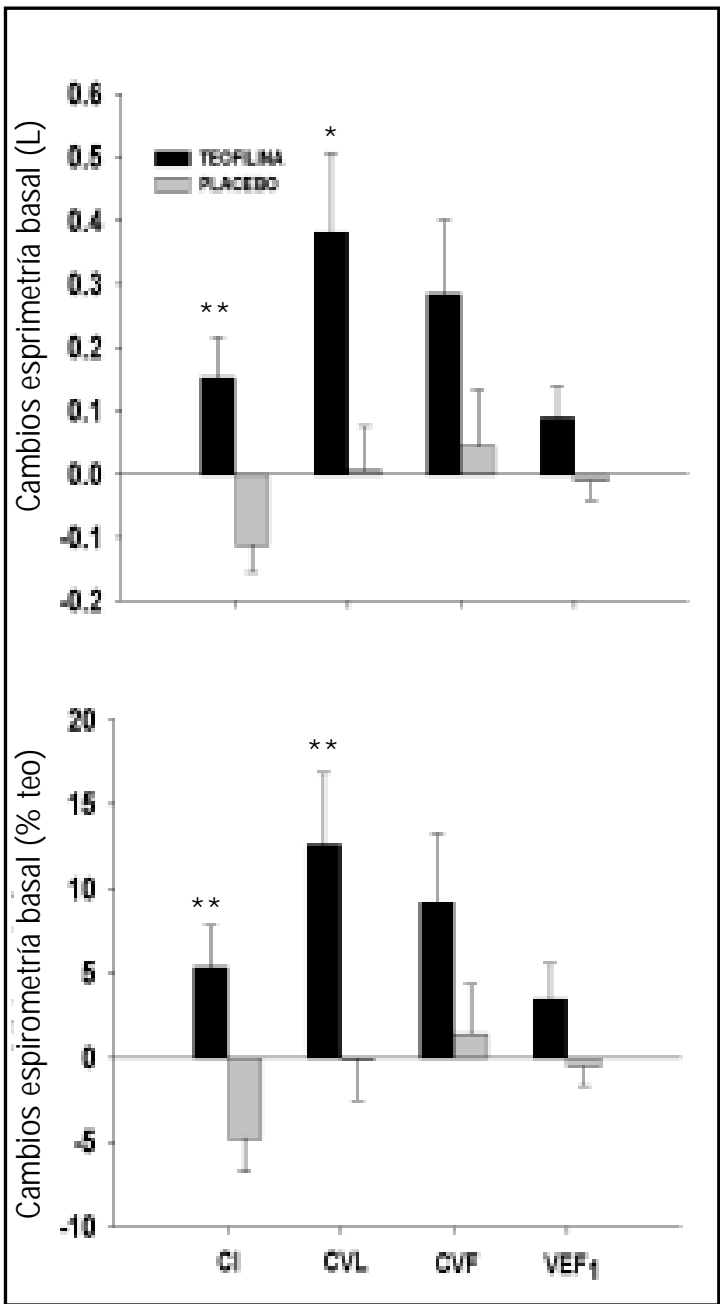

Figura 2. Panel superior: Cambios en los índices espirométricos pre-broncodilatador expresados en valores absolutos, observados en el grupo tratado con teofilina (barras negras) y con placebo (barras grises). Panel inferior: Cambios en los índices espirométricos pre-broncodilatador expresados en porcentaje del valor teórico en el grupo tratado con teofilina y con placebo. ${ }^{*}=p<0,05 ;{ }^{* *}=p<0,01$. tivamente mayor con teofilina (Tabla 2). No hubo cambios significativos en el $\mathrm{VEF}_{1}$ ni en la CVF. Los cambios espirométricos obtenidos después del broncodilatador fueron de muy pequeña magnitud. $\mathrm{Si}$ bien al comparar con placebo hubo aumentos estadísticamente significativos de CVL y $\mathrm{VEF}_{1}$, éstos carecieron de importancia clínica.

La PImax y la PEmax no cambiaron significativamente con teofilina, demostrando que no hubo efecto sobre la fuerza muscular respiratoria.

La proporción de pacientes que aumentó la DR6 sobre 30 metros, fue significativamente mayor al emplear teofilina (Tabla 2). Sin embargo, no

Tabla 2. Proporción de pacientes que superaron los límites de mejoría clínica previamente establecidos con la administración de teofilina y de placebo

\begin{tabular}{|lccl|}
\hline & Placebo & Teofilina & $\mathrm{p}$ \\
\hline CI & $0 / 19$ & $6 / 19$ & 0,008 \\
CVL & $3 / 19$ & $12 / 19$ & 0,003 \\
MRC & $4 / 19$ & $11 / 19$ & 0,02 \\
DR6 & $1 / 19$ & $8 / 19$ & 0,008 \\
C de Vida & $3 / 19$ & $11 / 19$ & 0,007 \\
\hline
\end{tabular}

Las fracciones corresponden al número de pacientes de ambos grupos que respondieron a cada uno de los cinco índices de mejoría clínica. CI=capacidad inspiratoria; $\mathrm{C}$ de Vida=calidad de vida. Para el resto de las abreviaturas ver Tabla 1.

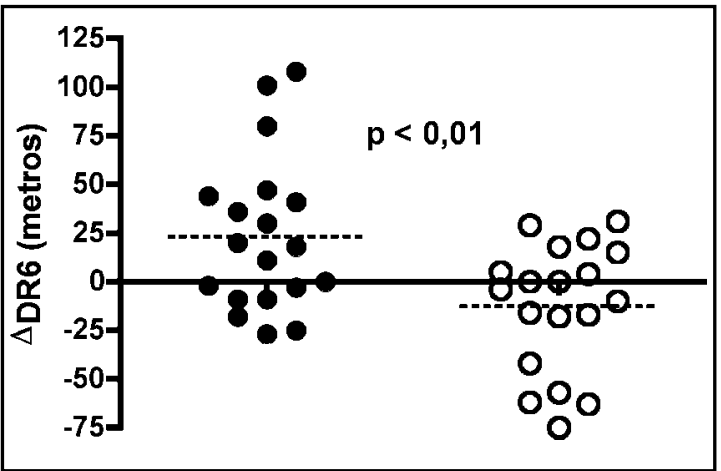

FIGURA 3. Cambios individuales de la distancia recorrida en 6 minutos ((DR6) en los pacientes que recibieron teofilina (círculos negros) y placebo (círculos vacíos). Las líneas horizontales discontinuas señalan los promedios de cada grupo. 
se observó diferencias significativas en el grado de disnea, fatiga, frecuencia cardíaca y $\mathrm{SpO}_{2}$ alcanzadas al final de la DR6 al comparar con placebo. La Figura 3 muestra los valores individuales del cambio en la DR6 en ambos grupos. Esta aumentó $23 \pm 40 \mathrm{~m}$ en los pacientes tratados con teofilina y disminuyó $13 \pm 33 \mathrm{~m}$ con placebo $(\mathrm{p}<0,01)$.

El índice de disnea disminuyó significativamente en $0,68 \pm 0,20$ puntos con teofilina y aumentó $0,11 \pm 0,18$ puntos con placebo $(p=0,042)$. La Figura 4 muestra en un gráfico de identidad los valores individuales del puntaje de disnea antes y después del tratamiento con teofilina y placebo.

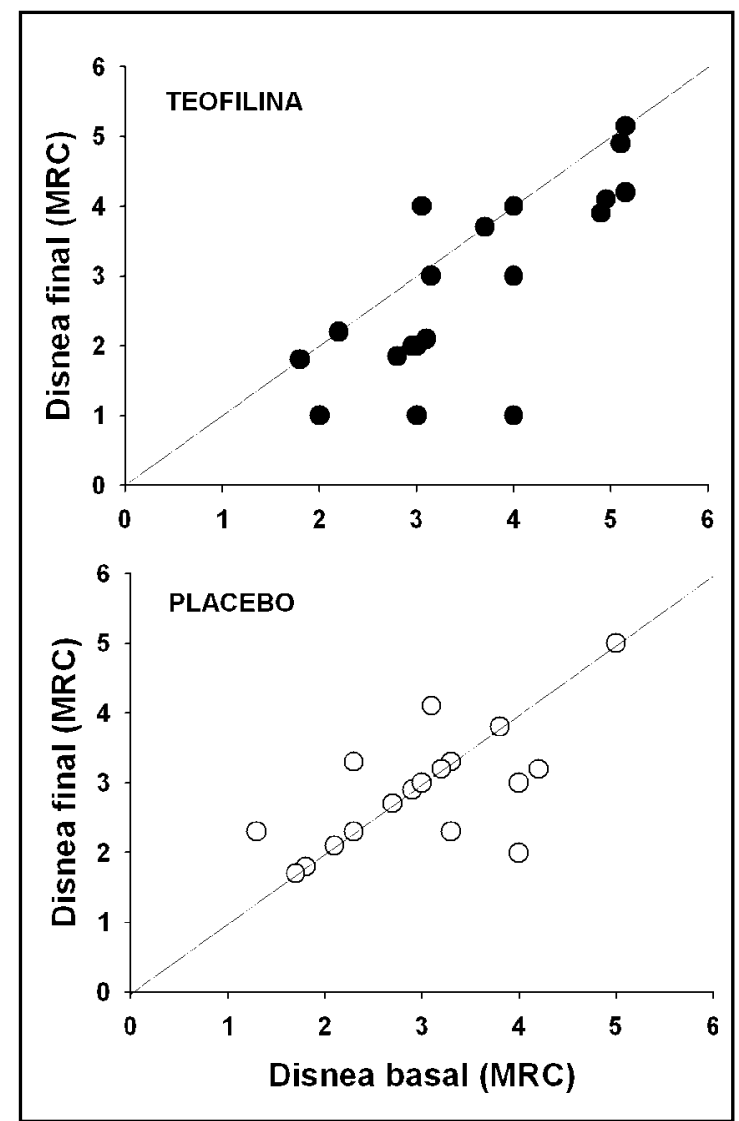

FiguRA 4. Gráficos de identidad que muestran el efecto sobre la disnea medido con el puntaje de la escala de disnea del British Medical Research Council (MRC) en cada individuo del grupo tratado (panel superior) y del grupo placebo (panel inferior). La línea diagonal corresponde a la línea de identidad. Cada punto corresponde a un paciente.
La proporción de pacientes que mejoró la disnea en un punto o más fue significativamente mayor con teofilina.

La teofilina, a diferencia del placebo, produjo una mejoría en el puntaje global de la calidad de vida $(p=0,011)$, y una proporción significativamente mayor de pacientes tratados superó el valor de 0,5 puntos considerado de importancia clínica. No se observó diferencias significativas en los cambios de los dominios correspondientes a disnea y aspecto emocional, mientras que hubo una mejoría significativa en el dominio de fatiga ( $p<0,04)$ y en el de la capacidad de control de la enfermedad $(p=0,03)$ con una proporción mayor de pacientes que sobrepasó los 0,5 puntos en el grupo tratado (Figura 5).

La Figura 6 ilustra la proporción de pacientes que no mejoraron o mejoraron 1,2 ó $\geq 3$ de los índices señalados en la Tabla 2 . Todos los pacientes presentaron beneficios con la teofilina y en 10 de ellos $(52,6 \%)$ se observó una mejoría en $\geq 3$ índices clínicos. En cambio, 10 de los 19 pacientes del grupo placebo no presentó ninguna mejoría. No fue posible identificar factores que explicaran la respuesta a un mayor número de parámetros en 10 de los 19 pacientes tratados con teofilina.

Los niveles plasmáticos de teofilina al $12^{\circ}$ día fueron variables y no se relacionaron con los cambios funcionales o clínicos observados.

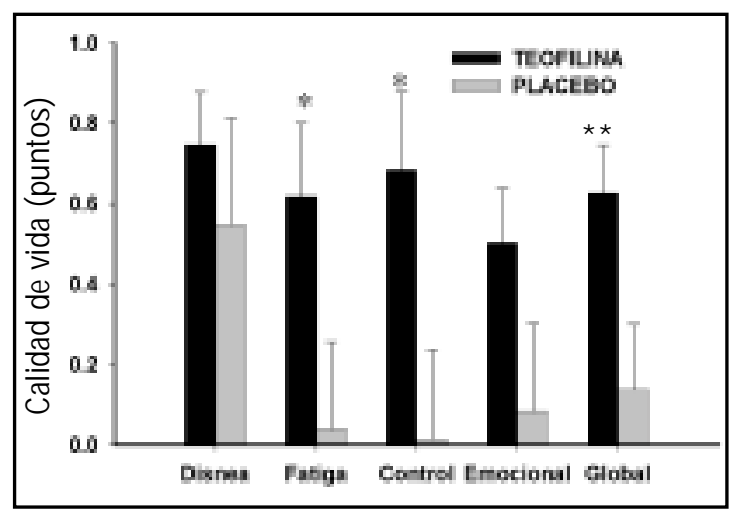

FIgURA 5. Efecto de teofilina y placebo sobre la calidad de vida global y en cada uno de los dominios. Control: capacidad de control de la enfermedad; Emocional: aspecto emocional. ${ }^{*}=p<0,05$; ${ }^{* *}=p<0,01$. 


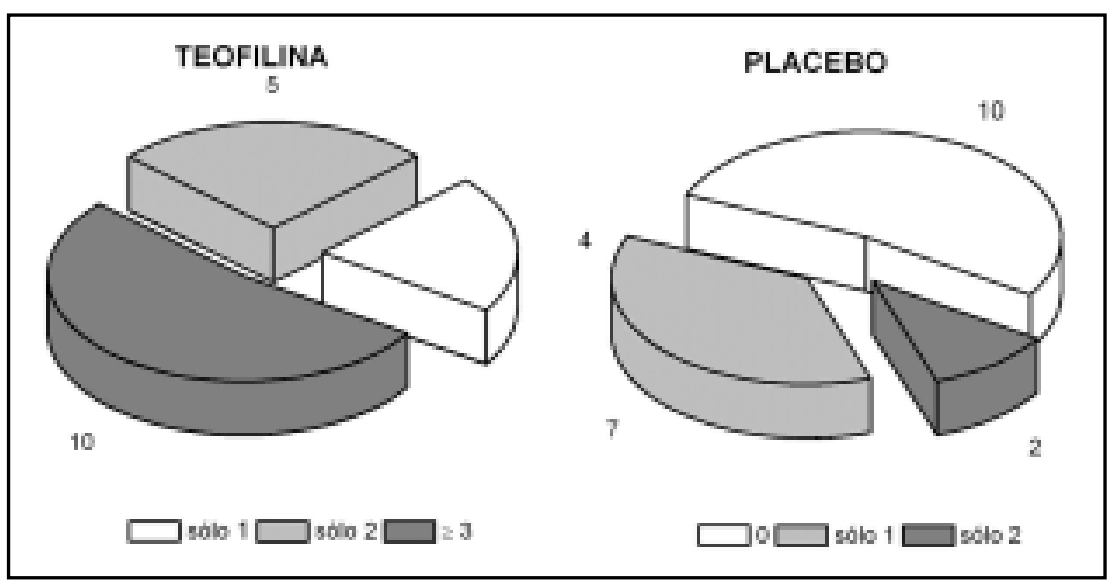

FiguRa 6. Número de pacientes que no mejoraron o mejoraron uno, dos $0 \geq 3$ índices clínicos con la administración de teofilina (figura izquierda) o con placebo (figura derecha). Se observa que todos los pacientes presentaron beneficios con la teofilina y que en 10 de ellos fue por mejoría en $\geq 3$ índices clínicos. Por el contrario, con placebo 10 de los 19 pacientes $(52,6 \%)$ no presentaron ninguna mejoría.

Cuatro pacientes tratados con teofilina, refirieron náuseas y dos de ellos presentaron vómitos. Los niveles plasmáticos de teofilina en estos pacientes fueron de 16,$9 ; 10,3 ; 9,1 ;$ y $21,8 \mathrm{mg} / \mathrm{l}$. Otro paciente, cuyo nivel plasmático alcanzó a $18,5 \mathrm{mg} / \mathrm{l}$, tuvo cefaleas frecuentes. En el grupo placebo, un paciente refirió náuseas, cefalea y anorexia y otro, mareos al incorporarse. La taquicardia como efecto colateral no fue señalada por ningún paciente, y la frecuencia cardíaca en reposo al final del estudio no fue mayor en el grupo tratado con teofilina $(84 \pm 13$ versus $77 \pm 12$ latidos/min).

Tres pacientes tratados con teofilina requirieron medicación de rescate en una oportunidad por mayor disnea y cuatro pacientes del grupo placebo debieron usar al menos una dosis diaria extra de $200 \mu \mathrm{g}$ de salbutamol durante todo el estudio. Otro enfermo requirió esta misma dosis en 8 oportunidades.

\section{DisCUSIÓN}

Los resultados del presente estudio demuestran que en pacientes con EPOC moderada a grave la adición de teofilina oral al tratamiento con salbutamol y bromuro de ipratropio en las dosis terapéuticas habituales tuvo los siguientes efectos: a) disminución de la hiperinflación pulmonar dinámica; b) alivio significativo de la disnea; c) aumento de la capacidad de ejercicio; y d) mejoría de la calidad de vida. Todos los pacientes presentaron mejoría en uno o más de los parámetros antes mencionados.

La disminución de la HPD con teofilina ha sido comunicada previamente por otros investigadores usando técnicas más complejas como la medición de volúmenes pulmonares con gases inertes y pletismografía ${ }^{12,13}$. También está descrita la ausencia de cambios del $\mathrm{VEF}_{1}$ y la CVF basales $4,11,12,28,29$, probablemente porque estas variables se relacionan más con los flujos espiratorios, los que no son modificados por broncodilatadores en la mayoría de los pacientes con EPOC a diferencia de lo que ocurre con los volúmenes pulmonares ${ }^{10}$.

Por otra parte, se ha comunicado que la teofilina alivia la disnea $5,12,15,18,28$, aunque empleando índices diferentes a la escala de MRC usada en el presente estudio, e incrementa la DR6 ${ }^{12,13}$. En nuestro estudio, este último efecto fue clínicamente relevante, pues superó el límite aceptado de 30 metros en la mayoría de los pacientes tratados con teofilina. Aunque se ha sugerido que el aumento de la capacidad de ejercicio es dosis-dependiente y relacionado con la disminución del aire atrapado ${ }^{12}$, no encontramos relación entre el aumento de la DR6 y la teofilinemia o el aumento de la CVL o la CI. 
La mejoría en la calidad de vida con teofilina se debió principalmente a una disminución de la fatiga y a una mayor capacidad de control de la enfermedad, a diferencia de lo comunicado por McKay y cols ${ }^{13}$ quienes encontraron además una mejoría en el dominio de disnea. En otros estu$\operatorname{dios}^{4,5}$ se ha observado que el efecto de la teofilina sobre la calidad de vida es semejante al que produce formoterol ${ }^{4}$ o salmeterol ${ }^{5}$ y que es significativamente mayor cuando teofilina se asocia a salmeterol ${ }^{5}$.

Un efecto aún controvertido de la teofilina es su acción sobre los músculos inspiratorios ${ }^{11,30,31}$. Para algunos autores, aumentaría la fuerza del diafragma y revertiría la fatiga de este músculo 11,30 . Nosotros no observamos cambios significativos en las presiones inspiratorias máximas, lo que concuerda con uno de estos estudios ${ }^{31}$.

El estudio de Nishimura y $\operatorname{cols}^{17}$ es similar al nuestro, con dosis semejantes de teofilina asociadas a la mezcla de salbutamol y bromuro de ipratropio. Observaron una mejoría significativamente mayor de la CVF, $\mathrm{VEF}_{1}$ y $\mathrm{PEF}$ con teofilina que con placebo, aunque sin diferencias en el puntaje de síntomas. Posteriormente, los mismos autores administraron dosis más elevadas de salbutamol y bromuro de ipratropio con la hipótesis de que al emplear una mayor dosis por vía inhalatoria, la teofilina no agregaría beneficios ${ }^{18}$. Sin embargo, el efecto fue similar al que habían obtenido previamente. En cambio, McKay y cols ${ }^{13}$ observaron que la teofilina en niveles plasmáticos de $17 \mathrm{mg} / \mathrm{l}$ asociada a salbutamol y bromuro de ipratropio, producía disminución del volumen de aire atrapado en el pulmón, alivio de la disnea y aumento de la DR6, efectos que no se observaron con niveles plasmáticos de $\sim 10 \mathrm{mg} / \mathrm{L}$. En el presente trabajo, sólo 8 de los 19 pacientes tuvieron niveles plasmáticos $\geq 17 \mathrm{mg} / \mathrm{l}$ y en tres éstos fueron inferiores a $10 \mathrm{mg} / \mathrm{l}$. Sin embargo, observamos en la mayoría de los enfermos mejorías significativas como grupo total.

Nuestros resultados coinciden con los de un metaanálisis reciente de Ram y cols ${ }^{32}$ sobre la eficacia de la teofilina en pacientes con EPOC estable. Los autores concluyen que la teofilina sigue siendo una opción importante en el tratamiento de estos pacientes, pues no sólo la prefieren al compararla con placebo sino que produce efectos benéficos objetivos en la función pulmonar, los gases arteriales y la capacidad ventilatoria. Los autores destacan, sin embargo, que es necesario pesar los beneficios en relación a los efectos adversos.

Dada la naturaleza de doble ciego de nuestro estudio, la teofilina se empleó en una dosis uniforme para todos los pacientes y sin titulación previa. A pesar de ello, la dosis elegida no difiere de la empleada en estudios en que se efectuó titulación para obtener los niveles plasmáticos deseados y evitar efectos adversos ${ }^{5,12}$. Los efectos adversos más frecuentes fueron los gastrointestinales que se presentaron en cuatro pacientes (21\%), porcentaje similar al observado por otros autores $4,29,32,33$. Se ha comunicado una frecuencia menor (11\%) cuando se titula la dosis ${ }^{5}$. Si bien consideramos que el número de pacientes de nuestro estudio es insuficiente para concluir que la dosis administrada carece de riesgos, es importante destacar que recientemente Ohta y $\operatorname{cols}^{34}$, observaron $5 \%$ de efectos adversos en 3.798 pacientes asmáticos o con EPOC mayores de 65 años y sólo fueron graves en 6 pacientes que tenían daño hepático o arritmias. Los niveles plasmáticos usando una dosis de $400 \mathrm{mg}$ diarios se midieron en 736 pacientes y en $87 \%$ fueron iguales o inferiores a $15 \mathrm{mg} / \mathrm{l}$. Según los autores, la teofilina es un medicamento que puede ser empleado sin riesgos en pacientes ancianos con asma o EPOC.

Junto a la ausencia de titulación de la teofilina ya señalada, también podría objetarse que el tratamiento broncodilatador inhalatorio empleado en nuestro estudio no fue necesariamente el mejor. Aunque el consenso de las Sociedades Americana y Europea de Enfermedades Respiratorias recomienda usar teofilina como terapia de la EPOC sólo si no ha habido respuesta a broncodilatadores de acción prolongada asociados a corticoides inhalatonios $^{35}$, estos últimos no tienen efecto demostrado sobre la hiperinflación pulmonar dinámica, la capacidad para caminar ni la disnea ${ }^{36}$. De hecho, la recomendación para usar corticoides inhalados se basa principalmente en que reduciría el número anual de exacerbaciones en pacientes con EPOC moderada a avanzada, efecto que no se evaluó en el presente estudio ${ }^{35,36}$. La razón de preferir utilizar una combinación de salbutamol y bromuro de ipratropio en lugar de emplear broncodilatadores de acción prolongada, como salmeterol, formoterol 
o tiotropio, fue su menor costo, buena tolerancia, su amplia disponibilidad en los servicios públicos de salud y, consecuentemente, su uso corriente en la práctica clínica.

En resumen, nuestros resultados demuestran que agregar teofilina de acción sostenida al tratamiento con salbutamol y bromuro de ipratropio proporciona beneficios funcionales y clínicos adicionales importantes que el paciente puede percibir, tales como mejoría de la disnea y de la calidad de vida, especialmente en el

\section{REFERENCIAS}

1. Pauwels RA, Buist AS, Calverley PMA, Jenkins CR. Global strategy for the diagnosis, management, and prevention of chronic obstructive pulmonary disease. NHLBI/WHO Global initiative for chronic obstructive lung disease (GOLD) workshop summary. Am J Respir Crit Care Med 2001; 163: 1256-76.

2. BaRnes PJ. Theophylline. New perspectives for an old drug. Am J Respir Crit Care Med 2003; 167: 813-8.

3. Mahier DA, Donohue JF, Barbee RA, Goldman MD, Gross NJ, WisNIEWSKI ME ET AL. Efficacy of salmeterol xinafoate in the treatment of COPD. Chest 1999; 115: 957-65.

4. Rossi A, Kristufek P, Levine BE, Thomson MH, Till D, Kottakis J et al. Comparison of the efficacy, tolerability, and safety of formoterol dry powder and oral slow release theophylline in the treatment of COPD. Chest 2002; 121: 1058-69.

5. Zuwallack RL, Mahler DA, Reily D, Church N, EMMETT A, Rickard $\mathrm{K}$ et al. Salmeterol plus theophylline combination in the treatment of COPD. Chest 2001; 119: 1661-70.

6. O’DonNell DE, WeBB KA. Exertional breathlessness in patients with chronic airflow limitation: the role of lung hyperinflation. Am Rev Respir Dis 1993; 148: 1351-7.

7. Díaz O, Viliafranca C, Ghezzo H, Borzone G, Leiva A, MiLC-EмLш J Eт AL. Role of inspiratory capacity on exercise tolerance in COPD patients with and without tidal expiratory flow limitation at rest. Eur Respir J 2000; 16: 269-75. aspecto relacionado con la capacidad para controlar la enfermedad. En consecuencia, parece razonable considerar su uso asociado a los broncodilatadores inhalados en los pacientes con EPOC más avanzada, siendo necesario evaluar su efecto individual, no sólo funcionalmente sino que también con indicadores clínicos simples y objetivos y, de acuerdo a ello, decidir su empleo prolongado. Idealmente debe medirse la teofilinemia para adecuar la dosis y evitar efectos no deseables.

8. Newton MF, O’DonNell DE, Forket L. Response of lung volumes to inhaled salbutamol in a large population of patients with severe hyperinflation. Chest 2002; 121: 1042-50.

9. Di Marco F, Milc-Emli J, Boveri B, Carlucci $P$, Santus P, CASANOVA F. Effect of inhaled bronchodilators on inspiratory capacity and dyspnoea at rest in COPD. Eur Respir J 2003; 21: 86-94.

10. Manríquez J, Díaz O, Borzone G, Lisboa C. Reversibilidad espirométrica en la enfermedad pulmonar obstructiva crónica. Efecto diferencial del salbutamol sobre el volumen espiratorio forzado del primer segundo y el volumen pulmonar. Rev Méd Chile 2004; 132: 787-93.

11. Murciano D, Auclair MH, Pariente R, Aubier M. A randomized, controlled trial of theophylline in patients with severe chronic obstructive pulmonary disease. N Engl J Med 1989; 320: 1521-5.

12. Chrystyn H, Muley B, Peake M. Dose response relation to oral theophylline in severe chronic obstructive airway disease. BMJ 1988; 297: 1505-10.

13. McKay SE, Howie CA, Thomson AH, Whiting B, AdDIs GJ. Value of teophylline treatment in patients handicapped by chronic obstructive lung disease. Thorax 1993; 48: 227-32.

14. TAylor DR, Buick B, Kinney C, Lowry RC, McDevitT DG. The efficacy of orally administered theophylline, inhaled salbutamol, and a combination of the two as chronic therapy in the management of chronic bronchitis with reversible air-flow obstruction. Am Rev Respir Dis 1985; 131: 747-51.

15. DuWNGer D, KRONENBerg R, Niewoehner DE. Efficacy of inhaled metaproterenol and orally-administered theophylline in patients with chronic airflow obstruction. Chest 1986; 89: 171-3. 
16. Tsukino M, Nishimura $K$, Ikeda A, Hajiro T, Koyama $\mathrm{H}$, Izum T. Effects of theophylline and ipratropium bromide on exercise performance in patients with stable chronic obstructive pulmonary disease. Thorax 1998; 53: 269-73.

17. Nishimura $K$, Koyama $H$, Ikeda $A$, Izumi $T$. Is oral theophylline effective in combination with both inhaled anticholinergic agent and inhaled B2 agonist in the treatment of stable COPD? Chest 1993; 104: 179-84.

18. Nishimura K, Koyoma H, Ikeda A, Sugiura N, KaWAKATSU $\mathrm{K}$, IzUm $\mathrm{T}$. The additive of theophylline on a high-dose combination of inhaled salbutamol and ipratropium bromide in stable COPD. Chest 1995; 107: 718-23.

19. American Thoracic Society. Standardization of spirometry: 1994 update. Am J Respir Crit Care Med 1995; 152: 1107-36.

20. Siafakas NM, Vermeire P, Pride NB, Paoletti P, Gibson J, Howard P. Optimal assessment and management of chronic obstructive pulmonary disease (COPD). Eur Respir J 1995; 8: 1398-420.

21. Black LF, HyatT RE. Maximal respiratory pressures. Normal values and relationship to age and sex. Am Rev Respir Dis 1969; 99: 696-702.

22. Butland RJA, Pang J, Gross ER, Woodcock AA, GEDDES DM. Two, six and 12 minute walking tests in respiratory diseases. BMJ 1982; 284: 1607-8

23. Borg GAV. Psychophysical basis of perceived exertion. Med Sci Sports Exerc 1982; 4: 377-81.

24. Redelmeir DA, Bayoumi AM, Goldstein RS, GuyatT $\mathrm{GH}$. Interpreting small differences in functional status: the six minute walk test in chronic lung disease patients. Am J Respir Crit Care Med 1997; 155: 1278-82.

25. Mahler DA, Wells C. Evaluation of clinical methods for rating dyspnea. Chest 1988; 93: 580-6.

26. Guell R, Casan P, Sangenis M, Sentis J, Morante F, BORRAS JM ET AL. Traducción española y validación de un cuestionario de calidad de vida en pacientes con enfermedad pulmonar obstructiva crónica. Arch Bronconeum 1995; 31: 202-10.

27. Jaeschke R, Singer AL, GuYATT GH. Measurement of health status: ascertaining the minimal clinically important difference. Control Clin Trials 1989; 10: 407-15.
28. Mahier DA, Matthay RA, Zinder PE, Wells CK, LOKE J. Sustained release theophylline reduces dyspnea in nonreversible obstructive airway disease. Am Rev Respir Dis 1985; 131: 22-5.

29. Vilianueva F, Bello S, Carrasco E, Sepúlveda R, Vicherat L, Barahona R et al. Caracterización de los pacientes portadores de enfermedad pulmonar obstructiva crónica y su respuesta a ipratropio y a teofilina. Rev Chil Enf Respir 1999; 15: 163-71.

30. Aubier M, De Troyer A, Sampson M, Mackiem PT, Roussos $\mathrm{CH}$. Amynophylline improves diaphragmatic contractility. NEng J Med 1981; 305: 249-52.

31. Мохнам J. Amynophylline and the respiratory muscles: An alternative view. Clin Chest Med 1988; 9: 325-40.

32. Ram FSF, Jardin JR, ATALah A, Castro AA, Mazzin $R$, GoLDSTEIN R ET AL. Efficacy of theophylline in people with stable chronic obstructive pulmonary disease: a systematic review and meta-analysis. Evidencebased review. Respir Med 2005; 99:135-44.

33. Belua V, Foresi A, Bianco S, Grassi V, Olvieri D, BeNSI $G$ et AL. Efficacy and safety of oxitropium bromide, theophylline and their combination in COPD patients: a double-blind ramdomized, multicentre study (BREATH TRIAL). Respir Med 2002; 96: 881-9.

34. Ohta K, Fukuchi Y, Grouse L, Mizutani R, Rabe KF, RENNARD S ET AL. A prospective clinical study of theophylline safety in 3810 elderly with asthma or COPD. Respir Med 2004; 98: 1016-24.

35. Ceш BR, MacNee W, Agusti A, Anzueto A, Berg B, Buist AS ET AL. Standards for the diagnosis and treatment of patients with COPD: a summary of the ATS/ERS position paper. Eur Respir J 2004; 23: 932-46.

36. Chronic Obstructive Pulmonary Disease. National clinical guideline on management of chronic obstructive pulmonary disease in adults in primary and secondary care. Thorax 2004; 59 (Suppl 1): i39-i130.

Agradecimientos

Los autores agradecen a Laboratorios SAVAL S.A. por el aporte de Elixine Lentocaps ${ }^{\circledR}$ y a Boehringer Ingelheim por el aporte de Combivent ${ }^{\circledR}$. 